\title{
Unifying framework for synchronization of coupled dynamical systems
}

\author{
S. Boccaletti, ${ }^{1,2}$ Louis M. Pecora, ${ }^{3}$ and A. Pelaez ${ }^{1}$ \\ ${ }^{1}$ Department of Physics and Applied Mathematics, Universidad de Navarra, Irunlarrea s/n, 31080 Pamplona, Spain \\ ${ }^{2}$ Istituto Nazionale di Ottica Applicata, Largo E. Fermi, 6, 50125 Florence, Italy \\ ${ }^{3}$ Code 6343, Naval Research Laboratory, Washington, DC, 20375
}

(Received 5 October 2000; revised manuscript received 21 February 2001; published 29 May 2001)

\begin{abstract}
A definition of synchronization of coupled dynamical systems is provided. We discuss how such a definition allows one to identify a unifying framework for synchronization of dynamical systems, and show how to encompass some of the different phenomena described so far in the context of synchronization of chaotic systems.
\end{abstract}

DOI: 10.1103/PhysRevE.63.066219

The word synchronization has a greek root " $\sigma \grave{v} \gamma \chi \rho o ́ v o s "$ " that means "to share the common time.", This original meaning has been maintained in the colloquial use of the word synchronization, as agreement or correlation in time of different processes [1]. While the study of synchronization phenomena in periodic dynamical systems has been active since the earlier days of physics, recently this phenomenon has been actively investigated in coupled chaotic systems. In this latter framework, many different synchronization features have been described so far, namely, complete or identical synchronization (IS) [2], phase (PS) [3] and lag (LS) synchronization [4], generalized synchronization (GS) [5], intermittent lag synchronization (ILS) $[4,6]$, and almost synchronization (AS) [7].

IS is the simplest form of synchronization and describes the interaction of two identical systems, leading to their trajectories remaining exactly in step with each other in the course of the time [2]. GS goes further in using completely different systems and associating the output of one system to a given function of the output of the other system [5]. PS is an intermediate regime characterized by the asymptotic boundedness of the phase difference of the two outputs, whereas the two chaotic amplitudes remain uncorrelated [3]. The relevance of PS for chaotic systems relies on the fact that a generic chaotic flow can be seen as composed of a small number of intrinsic modes of proper rotation, whose phases may be easily computed [8]. LS is an intermediate state between PS and IS, implying the asymptotic boundedness of the difference between the output of one system at time $t$ and the output of the other shifted in time of a lag time $\tau_{\text {lag }}$ [4]. ILS implies that the two systems are verifying LS most of the time, but intermittent bursts of local nonsynchronous behavior may occur $[4,6]$ in concomitance with the passage of the system trajectory into attractor regions with a local Lyapunov exponent different in sign from its global value $[4,6]$. Finally, AS results in the asymptotic boundedness of the difference between a subset of the variables of one system and the corresponding subset of variables of the other system [7].

The natural continuation of these pioneering works was to investigate synchronization phenomena in spatially extended or infinite dimensional systems [9], to test synchronization in experiments [10], and to investigate the mechanisms leading to destruction of synchronized states [11].
PACS number(s): 05.45.Xt, 05.45.Jn

However, in spite of such a plethora of theoretical studies and experimental verifications, there is still a lack of a unifying framework for synchronization of coupled dynamical systems. Recently Brown and Kocarev (BK) [12] made an interesting attempt to provide a general definition of synchronization, with the idea that there are different kinds of synchronization that might be captured in a single formalism. Their approach assumes a total system divisible into two subsystems in which one can define functions (properties) on each subsystem that are mappings from the space of trajectories and time to some Cartesian space. Mathematically this looks as follows: The total system is given by $z=[x, y], z$ $\in \mathbb{R}^{m_{1}+m_{2}}, x \in \mathbb{R}^{m_{1}}, y \in \mathbb{R}^{m_{2}}$, with each subsystem forming a trajectory $\phi_{x}\left(z_{0}\right)$ and $\phi_{y}\left(z_{0}\right)\left(z_{0}\right.$ being a generic initial condition) that are mapped by properties $g_{x}$ and $g_{y}$ to a new space $\mathbb{R}^{d}$. A function $h\left(g_{x}, g_{y}\right)$ on these trajectory properties is required with either $\|h\|=0$ or $\|h\| \rightarrow 0$ as $t \rightarrow \infty$. The choices of $g_{x}, g_{y}$ and $h$ determine the type of synchronization. The use of the trajectory spaces is necessitated since several definitions of synchronization need averages or integrals over (infinitely) long-time segments of system trajectories.

We will show that we can simplify and generalize the definition of synchronization to a more condensed and concrete form than the one above (we will not have to appeal to infinite dimensional trajectory spaces). Our approach will capture all the cases that the Brown and Kocarev approach does along with an entire class that their approach misses.

Let us assume for now that our system $\mathbf{Z} \in \mathbb{R}^{m}$ is divided into two subsystems, $\mathbf{X} \in \mathbb{R}^{m_{1}}$ and $\mathbf{Y} \in \mathbb{R}^{m_{2}}\left(m_{1}+m_{2}=m\right)$. Typically when one states that a system (say $y$ ) is synchronized to another $(x)$ one means that an event in $y$ always occurs when a particular event in $x$ occurs. Rulkov [13] has stated that synchronization means prediction of one system's values from another. One can identify events with points in the phase or state space of the subsystems and capture the notion of prediction by stating that there is a function from $\mathbf{X}$ to $\mathbf{Y}$ such that a particular point in $\mathbf{X}$ is mapped, uniquely, to one point in $\mathbf{Y}$. The mathematically rigorous definition of a function is adhered to here: one and only one point in the range for each point in the domain. However, we want our synchronization function to be realistic. Typically, when we search for evidence of synchronization in data or in numerical calculations we never have data that fall right on a given $\tilde{x}$ or on a given $\tilde{y}$. Rather, we have that the closer $x(t)$ is to $\tilde{x}$ the closer $y(t)$ is to $\tilde{y}$. The latter statement is captured rigorously by a continuous function; namely, the trajectories 


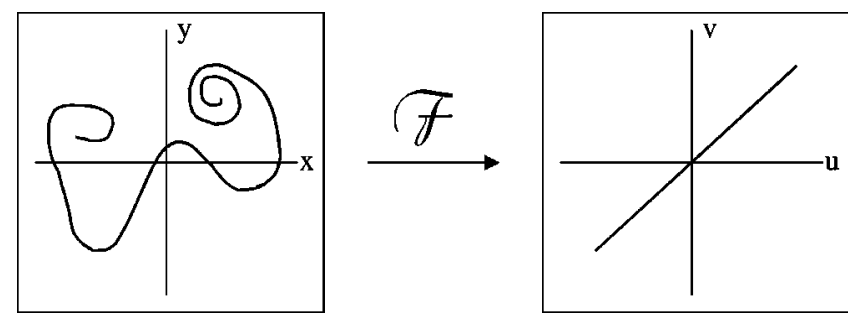

FIG. 1. The curved one-dimensional manifold in the twodimensional phase space $(x, y)$ is straightened out by the diffeomorphism $\mathcal{F}(z=(x, y)) \equiv(w=(u, v))$, so that the two projections of the new system $u=\mathcal{P}_{1}(w) \in \mathbf{U}, v=\mathcal{P}_{2}(w) \in \mathbf{V}$ form a synchronous state at any pair of points $(\tilde{u}, \tilde{v})$.

of $x(t)$ close to $\tilde{x}$ are mapped near to $\tilde{y}$ by a function that is continuous at the point $(\tilde{x}, \tilde{y})$. Note that we do not require smoothness in our function, allowing us to encompass more exotic relationships like generalized synchronization [5].

Before making the above into a rigorous mathematical statement, we use two examples to point out that one more stage is necessary. The first example is a curved onedimensional manifold in a two-dimensional phase space $\left(m_{1}=m_{2}=1\right)$, like the one shown in Fig. 1. Assume that the dynamics are on that manifold. In general there is no way one can have a continuous function from $x$ to $y$. However, suppose we consider a diffeomorphism $\mathcal{F}: \mathbf{Z} \rightarrow \mathbf{W}, w=\mathcal{F}(z)$ that "straightens out" the manifold. Then, if one considers the two projections of the transformed system $u=\mathcal{P}_{1}(w)$ $\in \mathbf{U}, v=\mathcal{P}_{2}(w) \in \mathbf{V}$, the new manifold in $u$ and $v$ forms a synchronization manifold and one can define a synchronous state at any pair of points $(\tilde{u}, \tilde{v})$. In fact, if the new manifold is a line at $45^{\circ}$ to the $u$ axis, one can even identify the continuous function from $u \rightarrow v$ : the identity. We note that such a transformation is not possible in the BK formalism, although it can be inserted as a preliminary step. The second example is suggested by tests for PS between two subsystems $x$ and $y$. In some cases, phase information can be acquired by the help of the Hilbert transform [14] of the components of $z, \quad r_{i}(t)=(1 / \pi) \int_{-\infty}^{+\infty} 1 /\left(t^{\prime}-t\right) \Phi_{i}\left(t^{\prime}\right.$ $-t, z(t)) d t^{\prime}$, where $\Phi_{i}\left(t^{\prime}, z(t)\right)$ is the $i$ th component of the flow causing $z(t) \rightarrow z\left(t+t^{\prime}\right)$. The phases are here generated as "new coordinates" $u_{i}(t)=\arctan \left[r_{i}(t) / x_{i}(t)\right]$ and $v_{i}(t)=\arctan \left[r_{i+m_{1}}(t) / y_{i}(t)\right]$, and can be compared for a given $i$ (we assume for now that $m_{1}=m_{2}$ ). What is important to note is that the transformation from $z \rightarrow r \rightarrow(u, v)$ requires an integration over the trajectory $z(t)$, but it does not require a function from the space of trajectories to $\mathbb{R}$. Rather, the expression used is just a function from $z(t) \rightarrow r(t)$, point to point; it is the flow function that allows us to avoid the more abstract and complex trajectory space. The use of the flow function must be done first on the entire trajectory $z(t)$ and cannot be done separately on components since, in general, the components are coupled and each one's dynamics depends on the other. Finally, we note that we could include any BK types of property functions $g_{u}$ and $g_{v}$ (with appropriate modifications) using a flow and then a general transformation $g=\left(g_{u}, g_{v}\right)$ that splits into the two properties. An important point to highlight is that, for some applications, we may only need to compare a subset of the new coordinates $u$ and $v$ from each system. Therefore, in the following we will denote by $\mathcal{F}: \mathbb{R}^{m_{1}+m_{2}} \rightarrow \mathrm{R}^{d_{1}+d_{2}}$ a function that, after application of the diffeomorphism, extracts $d_{1}\left(d_{2}\right)$ components out of the first $m_{1}$ (the second $m_{2}$ ) coordinates of the transformed space.

We now have all the main features we need to construct a more rigorous and very general definition of synchronization: (1) a function from the original phase space $z$ to a new phase space $(u, v),(2)$ the projections $\mathcal{P}_{1}$ and $\mathcal{P}_{2}$ onto the components of the new space, and (3) the synchronization relation, a continuous function.

Let us start by refining the definition of the continuous function to include consistency with the dynamics.

Definition. A function $f$ is a synchronization function at $(\tilde{u}, \tilde{v})$ if (a) $\tilde{v}=f(\tilde{u})$, (b) it is continuous at $\tilde{u}$, and (c) it is consistent with the dynamics $(u(t), v(t))$ locally, that is, if $\delta$ and $\epsilon$ are a valid pair for the continuity property $[|u-\tilde{u}|$ $<\delta$ implies $|f(u)-\tilde{v}|<\epsilon]$, then the dynamics is such that if $|u(t)-\tilde{u}|<\delta$ we have $|v(t)-\tilde{v}|<\epsilon$.

In words, near $(\tilde{u}, \tilde{v})$ the function describes well the predictability of subsystem $\mathbf{V}$ dynamics from subsystem $\mathbf{U}$ dynamics. Now for the sake of rigor we pay closer attention to such details as initial conditions and time. For the time being, let $\mathcal{B}$ be the basin of attraction for the attractor $\mathcal{A}$ of a dynamical system $\mathbf{Z} \subset \mathbb{R}^{m}$. Let $\mathcal{P}_{1}$ and $\mathcal{P}_{2}$ be projections from $\mathbb{R}^{d_{1}+d_{2}}$ to $\mathbb{R}^{d_{1}}$ and to $\mathbb{R}^{d_{2}}$, respectively.

Definition. For a given function $\mathcal{F}: \mathbb{R}^{m} \rightarrow \mathbb{R}^{d_{1}+d_{2}}$, a dynamical system $\mathbf{Z} \subset \mathbb{R}^{m}$ contains locally synchronous subsystems in $\tilde{z} \in \mathcal{A}$ if $\forall \quad z_{0} \in \mathcal{B}$ there is a time $T$ such that for $t \geqslant T$ a synchronization function exists at $\left(\tilde{u}=\mathcal{P}_{1}(\mathcal{F}(\tilde{z})), \tilde{v}\right.$ $\left.=\mathcal{P}_{2}(\mathcal{F}(\tilde{z}))\right)$.

We can think of the subsystems as having properties $u$ and $v$ that are synchronous only near the part of the trajectory, assuming the trajectory comes close to $\tilde{u}$. We cannot say what the relationship is between $u(t)$ and $v(t)$ anywhere else on the trajectory. The nature of the synchronization function $f$ and the function $\mathcal{F}$ determine the type of synchronization we are considering.

The above definition is a local definition (that is, it refers explicitly to a given position $\tilde{z} \in \mathcal{A}$ ). While extension of such a definition for global synchronization will be provided in the following, at the present stage it is important to remark that such a feature is crucial if one wants to describe phenomena such as ILS, which is, indeed, intimately related to the local stability properties of the flow on the chaotic attractor.

We now discuss how to extend the definition to the entire trajectory of the transformed system $w(t)$ so as to have a single continuous function everywhere on the image of the attractor under $\mathcal{F}$. This might seem to be just a matter of having enough points of local synchronization, but there are two things to consider carefully. One is that we want to make sure every point on the trajectory is mapped by a synchronization function between the two subsystems $u$ and $v$. The other is more subtle. We would like there to be one continuous function on the whole trajectory or attractor. As our defi- 
nition stands, generally, we have a function associated with each synchronization pair $(\tilde{u}, \tilde{v})$, but the functions may be different in their local continuity. The valid $\delta$ and $\epsilon$ pairs need not have any particular relationship between different synchronization functions.

A single function that maps the $U$ projection of $\mathcal{F}(\mathcal{A})$ $\left[\mathcal{P}_{1}(\mathcal{F}(\mathcal{A}))=\mathbf{U}\right]$ to the $V$ projection of $\mathcal{F}(\mathcal{A})\left[\mathcal{P}_{2}(\mathcal{F}(\mathcal{A}))\right.$ $=\mathbf{V}]$ can be attained, and we will show this in the theorem below. The essential feature we need to add is a set of synchronization points on the attractor that provide a "covering" property.

Definition. If $\left\{u_{i}\right\}$ is a set of points on $\mathbf{U}$ and $\left\{f_{i}\right\}$ is a set of continuous functions, one associated with each $u_{i}$, from $\mathbf{U}$ to $\mathbf{V}$, then the functions provide a continuity covering of $\mathbf{U}$ if $\forall \varepsilon>0$ the set of all valid $\delta_{i}$ 's associated with $\varepsilon$ [one for each $\left(u_{i}, f_{i}\right)$ pair] covers the set $\mathbf{U}$.

This gives the following theorem that provides the unique synchronization function.

Theorem. If the subsystem $\mathbf{U}$ contains a set of synchronization points $\left\{u_{i}\right\}$ and the associated functions $\left\{f_{i}\right\}$ provide a continuity covering of $\mathbf{U}$, then there exists a unique, global, continuous synchronization function $f: \mathbf{U} \rightarrow \mathbf{V}$.

Proof. Let us proceed by absurdity, and suppose there exist two different realizations of the dynamics $z_{1} \in \mathcal{A}$ and $z_{2} \in \mathcal{A}$ such that $\mathcal{P}_{1}\left(\mathcal{F}\left(z_{1}\right)\right)=\mathcal{P}_{1}\left(\mathcal{F}\left(z_{2}\right)\right)=u, \quad \mathcal{P}_{2}\left(\mathcal{F}\left(z_{1}\right)\right)$ $=v_{1}$, and $\mathcal{P}_{2}\left(\mathcal{F}\left(z_{2}\right)\right)=v_{2}$. Let $\eta=\left|v_{1}-v_{2}\right|$ be the distance between the two images of $z_{1}$ and $z_{2}$ in $\mathbb{R}^{d_{2}}$, and pick $\varepsilon$ $<\eta / 2$. Let $u_{k}$ be the synchronization point whose neighborhood of radius $\delta_{k}(\varepsilon)$ contains $u$ (its existence is guaranteed by the continuity covering property), and let $f_{k}$ be the associated synchronization function. Because of the consistency of $f_{k}$ with the dynamics, we must have $\left|f_{k}\left(u_{k}\right)-v_{1}\right|<\varepsilon$ and $\left|f_{k}\left(u_{k}\right)-v_{2}\right|<\varepsilon$. Adding the latter two inequalities and using the triangular inequality we have $\left|v_{1}-v_{2}\right|<\eta$, which contradicts our hypothesis. Thence, there exists a function $f$ mapping all points $u \in \mathbf{U}$ into the corresponding points $v$ $=f(u) \in \mathbf{V}$.

Next we show that $f$ is continuous at all points $u \in \mathbf{U}$. For all $\varepsilon / 2$ there is a $\delta_{j}$ associated with one of the synchronization points $u_{j}$ such that $\left|u-u_{j}\right|<\delta_{j}$. Pick $\delta>0$ so that the set $S_{\delta}=\left\{u^{\prime}:\left|u^{\prime}-u\right|<\delta\right\}$ is completely contained in the set of points within $\delta_{j}$ around $u_{j}$. Because of consistency of $f_{j}$ with the dynamics $\forall u^{\prime} \in S_{\delta}$ we must have $\mid f\left(u^{\prime}\right)$ $-f_{j}\left(u_{j}\right) \mid<\varepsilon / 2$. On the other hand, we also have $\mid f_{j}\left(u_{j}\right)$ $-f(u) \mid<\varepsilon / 2$. Using again the triangular inequality we have $\left|f\left(u^{\prime}\right)-f(u)\right|<\varepsilon$ whenever $\left|u^{\prime}-u\right|<\delta$.

The above provides a definition of perfect synchronization. In many realistic applications, however, one must account for noise, or for a finite measurement resolution, so that it is useful to introduce a fuzziness parameter, setting up the minimal coarsening scale at which the states of one projected set may be put in correspondence with the states of the other projected set.

Definition. For a given function $\mathcal{F}: \mathbb{R}^{m} \rightarrow \mathbb{R}^{d_{1}+d_{2}}$, a dynamical system $\mathbf{Z}$ contains locally $\sigma$-synchronous subsystems in $\tilde{z} \in \mathcal{A}$ if $\forall z_{0} \in \mathcal{B}$ there is a time $T$ such that $\forall \varepsilon>\sigma \exists \delta>0$ such that $t \geqslant T$ and $\mid \mathcal{P}_{1}\left(\mathcal{F}\left(\Phi\left(t, z_{0}\right)\right)\right)$ $-\mathcal{P}_{1}(\mathcal{F}(\tilde{z}))|<\delta \Rightarrow| \mathcal{P}_{2}\left(\mathcal{F}\left(\Phi\left(t, z_{0}\right)\right)\right)-\mathcal{P}_{2}(\mathcal{F}(\tilde{z})) \mid<\varepsilon$.
This last definition recovers the previous one for $\sigma \rightarrow 0$, and is tantamount to saying that the consistency of the synchronization function $f$ with the dynamics holds only up to a minimum scale $\sigma$, giving the minimal coarsening or precision scale for which states in one subspace may be put in correspondence with states in the other subspace. Although the value of $\sigma$ is not constrained in our definition, we should point out that if $\sigma$ is larger than the diameter of $\mathcal{P}_{2}(\mathcal{F}(\mathcal{A})$ ) then the above is trivially satisfied $\forall \delta>0$ and $\forall z_{0} \in \mathcal{B}$. Therefore, the only relevant cases are the ones in which $\sigma$ is considerably smaller than the diameter of $\mathcal{P}_{2}(\mathcal{F}(\mathcal{A}))$.

Furthermore, global $\sigma$ synchronization may be described as the case for which local $\sigma$-synchronization features are displayed independently of the particular choice of $\tilde{z} \in \mathcal{A}$.

With the help of what is discussed above, let us move to show some examples of synchronization phenomena that can be encompassed within our definition.

Generalized and identical synchronization. In Refs. [2,5], GS and IS are characterized by the fact that the asymptotic evolution of the system occurs within a manifold defined by $y=K(x), K$ being a generic function (IS is the case for which $m_{1}=m_{2}$ and $K$ coincides with the identity). In our framework, GS can be considered as a particular case of global synchronization with $\sigma=0$, by setting $u=x$ and $v$ $=y=K(x)$, so as the synchronization function comes out to be $f=K$.

Phase synchronization. PS consists in a collective evolution of a pair of weakly coupled chaotic systems characterized by a phase distance that is asymptotically bounded around a constant value $c$, whereas the amplitudes may remain uncorrelated [3]. We assume the above situation is described by two proper phase functions $\psi(x(t))$ and $\psi(y(t))$, whose outputs are time dependent scalar quantities ranging from 0 to $2 \pi$, that are chosen so as $|\psi(x)-\psi(y)|<R$, where $R$ is the size of the residual fluctuations of the phase distance around $c$. Let $\mathcal{F}$ be the function having components $\psi(x), \psi(y) \quad\left(d_{1}=d_{2}=1\right)$. What we have to show is that $\forall \varepsilon>\sigma \exists \delta>0$ such that

$$
|\psi(x)-\psi(\tilde{x})|<\delta \Rightarrow|\psi(y)-\psi(\tilde{y})|<\varepsilon, \forall \quad \tilde{z} \equiv(\tilde{x}, \tilde{y}) .
$$

Now,

$$
\begin{aligned}
|\psi(y)-\psi(\tilde{y})| & =|\psi(y)-\psi(x)+\psi(x)-\psi(\tilde{y})+\psi(\tilde{x})-\psi(\tilde{x})| \\
\leqslant & |\psi(y)-\psi(x)|+|\psi(\tilde{x})-\psi(\tilde{y})| \\
& +|\psi(x)-\psi(\tilde{x})| \\
< & 2 R+|\psi(x)-\psi(\tilde{x})| .
\end{aligned}
$$

Therefore, selecting $\sigma=2 R$ and $\delta=\varepsilon-2 R$, the definition of global $\sigma$ synchronization is satisfied.

Lag and almost synchronization. LS refers to a case in which asymptotically $\left|x(t)-y\left(t-\tau_{\text {lag }}\right)\right|<R$, for a given lag time $\tau_{\text {lag }}$ [4]. LS can be identified with a global $\sigma$-synchronization phenomenon. Now with $z=(x, y)$ define the transformation $\mathcal{F}$ such that 


$$
\begin{aligned}
u & =\mathcal{P}_{1}(\mathcal{F}(z))=x \quad \text { and } \quad v=\mathcal{P}_{2}(\mathcal{F}(z))=\mathcal{P}_{2}(\Phi(-\tau, z)) \\
& =y(t-\tau) .
\end{aligned}
$$

Then

$$
|v(t)-u(t)|<R
$$

and

$$
\begin{aligned}
\left|v_{1}(t)-v_{2}(t)\right|= & \mid v_{1}(t)-u_{1}(t)+u_{2}(t)-v_{2}(t)+u_{1}(t) \\
& -u_{2}(t) \mid \\
\leqslant & \left|v_{1}(t)-u_{1}(t)\right|+\left|u_{2}(t)-v_{2}(t)\right|+\mid u_{1}(t) \\
& \quad-u_{2}(t) \mid \\
< & 2 R+\left|u_{1}(t)-u_{2}(t)\right| .
\end{aligned}
$$

$\sigma=2 R$ and $\delta(\varepsilon)=\varepsilon-2 R$ satisfy our definition of global $\sigma$ synchronization and when $R=0$ we get the exact case of a synchronization function $f: u \rightarrow v$, where, in this case, $f$ is just the identity.

AS corresponds to a situation where asymptotically $\left|\mathcal{P}_{l}(x(t))-\mathcal{P}_{l}(y(t))\right|<R$, with $l<m_{1}, m_{2}$ and $\mathcal{P}_{l}$ being the projector extracting the first $l$ components out of the vectors $x$ and $y$ [7]. The same demonstration used for LS holds for $\sigma=2 R, \quad \delta=\varepsilon-2 R$, and $\mathcal{F}_{i}=z_{i}, i=1, \ldots, l ; \mathcal{F}_{i}=z_{i+m_{1}-l}, i$ $=l+1, \ldots, 2 l$.

Intermittent phenomena. We finally discuss how ILS can be embraced in our framework of synchronization. We first highlight that ILS is a local phenomenon, in the sense that it depends explicitly on the local stability property of the flow onto the chaotic attractor $[4,6]$. However, since ILS has an intermittent nature, in the following we have to extend our definition.

Definition. For a given function $\mathcal{F}: \mathbb{R}^{m} \rightarrow \mathbb{R}^{d_{1}+d_{2}}$, a dynamical system contains intermittently $\sigma$-synchronous subsystems $\left\{\widetilde{z}_{i}^{0}\right\} \subset \mathcal{A}$ if $\forall z_{0} \in \mathcal{B}$ there is a time $T_{i}$ and a time interval $\Delta T_{i}$ associated with each $z_{i}$ such that $\forall \epsilon$ $>\sigma \exists \delta>0$ such that $\forall t_{1}>T_{i} \exists t_{2}>t_{1}$ for which $t_{2}$ $\leqslant t \leqslant t_{2}+\Delta T_{i}$ and

$$
\begin{aligned}
& \left|\mathcal{P}_{1}\left(\mathcal{F}\left(\Phi\left(t, z_{0}\right)\right)\right)-\mathcal{P}_{1}\left(\mathcal{F}\left(\Phi\left(t-t_{2}, \widetilde{z}_{i}^{0}\right)\right)\right)\right| \\
& \quad<\delta \Rightarrow\left|\mathcal{P}_{2}\left(\mathcal{F}\left(\Phi\left(t, z_{0}\right)\right)\right)-\mathcal{P}_{2}\left(\mathcal{F}\left(\Phi\left(t-t_{2}, \widetilde{z}_{i}^{0}\right)\right)\right)\right| \\
& \quad<\varepsilon
\end{aligned}
$$

In the above we have a special case of our local $\sigma$ synchronization in that we have a continuous infinity of synchronous points $\tilde{z}_{i}(t)$ emerging from each $\widetilde{z}_{i}^{0}$ and reached in order using the flow $\Phi$, but only over a finite interval of time $\Delta T_{i}$. In the limit $\sigma \rightarrow 0$ we obtain local functions $f_{i}$ that map $u$ $=\mathcal{P}_{1}\left(\mathcal{F}\left(\Phi\left(t, z_{0}\right)\right)\right)$ to $v=\mathcal{P}_{2}\left(\mathcal{F}\left(\Phi\left(t, z_{0}\right)\right)\right)$ in the appropriate time intervals.

The authors are indebted to F. T. Arecchi, S. Bressler, R. Brown, T. Carroll, L. Kocarev, R. Livi, H. Mancini, U. Parlitz, A. Pikovsky, A. Politi, I. Procaccia, M. Rabinovich, N. Rulkov, T. Sauer, S. Schiff, and D. L. Valladares for many fruitful discussions. S.B. acknowledges financial support from EU Contract No. ERBFMBICT983466.
[1] A.S. Hornby, Oxford Advanced Dictionary (Oxford University Press, 1974).

[2] H. Fujisaka and T. Yamada, Prog. Theor. Phys. 69, 32 (1983); V.S. Afraimovich, N.N. Verichev, and M.I. Rabinovich, Izv. Vyssh. Uchebn. Zaved., Radiofiz. 29(9), 1050 (1986); L.M. Pecora and T.L. Carroll, Phys. Rev. Lett. 64, 821 (1990).

[3] M.G. Rosenblum, A.S. Pikovsky, and J. Kurths, Phys. Rev. Lett. 76, 1804 (1996); E.R. Rosa, E. Ott, and M.H. Hess, ibid. 80, 1642 (1998).

[4] M.G. Rosenblum, A.S. Pikovsky, and J. Kurths, Phys. Rev. Lett. 78, 4193 (1997).

[5] N.F. Rulkov, M.M. Sushchik, L.S. Tsimring, and H.D.I. Abarbanel, Phys. Rev. E 51, 980 (1995); L. Kocarev and U. Parlitz, Phys. Rev. Lett. 76, 1816 (1996).

[6] S. Boccaletti and D.L. Valladares, Phys. Rev. E 62, 7497 (2000).

[7] R. Femat and G. Solis-Perales, Phys. Lett. A 262, 50 (1999).

[8] T. Yalçinkaya and Y.-C. Lai, Phys. Rev. Lett. 79, 3885 (1997).

[9] D.H. Zanette, Phys. Rev. E 55, 5315 (1997); P. Parmananda, ibid. 56, 1595 (1997); A. Amengual, E. Hernández-García, R. Montagne, and M. San Miguel, Phys. Rev. Lett. 78, 4379 (1997); S. Boccaletti, J. Bragard, F.T. Arecchi, and H.L. Mancini, ibid. 83, 536 (1999); H. Chaté, A. Pikovsky, and O.
Rudzick, Physica D 131, 17 (1999); S. Boccaletti, D.L. Valladares, J. Kurths, D. Maza, and H. Mancini, Phys. Rev. E 61, 3712 (2000).

[10] C. Schafer, M.G. Rosenblum, J. Kurths, and H.H. Abel, Nature (London) 392, 239 (1998); P. Tass, M.G. Rosenblum, M.G. Weule, J. Kurths, A. Pikovsky, J. Volkmann, A. Schnitzler, and H.J. Freund, Phys. Rev. Lett. 81, 3291 (1998); G.D. Van Wiggeren and R. Roy, Science 279, 1198 (1998); A. Neiman, X. Pei, D. Russell, W. Wojtenek, L. Wilkens, F. Moss, H.A. Braun, M.T. Huber, and K. Voigt, Phys. Rev. Lett. 82, 660 (1999); G.M. Hall, S. Bahar, and D.J. Gauthier, ibid. 82, 2995 (1999); A. R. Yehia, D. Jeandupeux, F. Alonso, and M.R. Guevara, Chaos 9, 916 (1999); J.-W. Shuai and D.M. Durand, Phys. Lett. A 264, 289 (1999); C.M. Ticos, E. Rosa, Jr., W.B. Pardo, J.A. Walkenstein, and M. Monti, Phys. Rev. Lett. 85, 2929 (2000).

[11] E. Barreto, P. So, B.J. Gluckman, and S.J. Schiff, Phys. Rev. Lett. 84, 1689 (2000); E. Barreto and P. So, ibid. 85, 2490 (2000).

[12] R. Brown and L. Kocarev, Chaos 10, 344 (2000).

[13] N.F. Rulkov (private communication).

[14] D. Gabor, J. IEEE London 93, 429 (1946). 